Using the DETECT PAH calculator, 14/ 41 (34.14\%) were recommended for RH as follows: an initial value above 300 points in STEP 1 (with further indication of echocardiographic assessment) and subsequent STEP 2 over 35 were recorded. On the other hand, only $9 / 41(21.95 \%)$ cases met the ultrasound criteria to be addressed to an invasive assessment when applying the ESC/ERS 2009 guidelines, the difference being statistically significant as compared to DETECT $(p<0.05)$.

PAH was finally confirmed in $8 / 41(19.51 \%)$ cases, supporting our preliminary results in a pilot study on a limited number of patients.

Conclusions: DETECT is a reliable algorithm for early detection of PAH in patients with SSc, optimizing PAH screening and prompting the rheumatologist-cardiologist collaboration.

Disclosure of Interest: None declared

DOI: 10.1136/annrheumdis-2017-eular.4053

\section{FRI0384 A MINI-INVASIVE TECHNIQUE FOR HAEMODYNAMIC EVALUATION: NEW PERSPECTIVES FOR PULMONARY ARTERIAL HYPERTENSION (PAH) DIAGNOSIS IN SYSTEMIC SCLEROSIS (SSC)}

E. Bellucci ${ }^{1}$, M.S. Romano ${ }^{2}$, M. Chiostri ${ }^{2}$, C. Bruni ${ }^{1}$, C. Giglioli ${ }^{2}$, P. Bernardo ${ }^{3}$, M.L. Conforti ${ }^{1}$, S. Bellando-Randone ${ }^{1}$, S. Guiducci ${ }^{1}$, M. Matucci-Cerinic ${ }^{1}$.

${ }^{1}$ Department of Experimental and Clinical Medicine, Division of Rheumatology, University of Florence; ${ }^{2}$ Department of Heart and Vessels, Division of Cardiology I: ${ }^{3}$ Department of Heart and Vessels, Division of Structural Interventional Cardiology, Azienda Ospedaliera Universitaria Careggi, Firenze, Italy

Background: PAH is one of the most severe complication and cause of mortality in SSc, with frequent late diagnosis as asymptomatic in its early stages. Right Heart catheterization $(\mathrm{RHC})$ is the gold standard for $\mathrm{PAH}$ detection, though its invasive nature with high risk of procedure related complications stress the need for developing new screening methods to investigate it in early and possibly reversible stages. Pressure Recording Analytical Method (PRAM) was recently developed to obtain a minimally invasive haemodynamic monitoring, using an arterial line and proximal pulsossimetry. This analyses arterial blood pressure curve and measure both cardiac [Cardiac Index, Cardiac Contractility and Vascular Stiffness ratio $(\mathrm{dP} / \mathrm{dT})$ ] and vascular [vascular stiffness, elastance], which can be usually obtained only through $\mathrm{RHC}$.

Objectives: to evaluate the clinical usefulness of PRAM method in SSc patients and to identify haemodynamic parameters related to increased risk of developing $\mathrm{PAH}$.

Methods: 40 ssc patients ( 35 women, mean age $60 \pm 9$,3 years; mean disease duration 7.5 years) were evaluated with both RHC and PRAM on the same day. Mean pulmonary arterial pressure (mPAP), cardiac index $(\mathrm{Cl})$, systemic vascular resistances (SVR), right cardiac power index (RCPI, calculated with mPAPXCl PRAM/451) were measured and concordance of the two methods was

\begin{tabular}{lccc}
\hline Univariate Logistic Regression & Unadjusted OR & $95 \% \mathrm{Cl}$ & $\mathrm{p}$ value \\
\hline Dicrote Pressure & 1.04 & $1.00-1.09$ & 0.064 \\
Arterial Elastance & 3.16 & $0.70-14.24$ & 0.134 \\
Right Cardiac Power index & 1.11 & $1.04-1.18$ & 0.002 \\
DLCO & 0.94 & $0.90-0.99$ & 0.021 \\
Multivariate Logistic regression with & & & \\
$\quad$ backward stepwise analysis & Unadjusted OR & $95 \% \mathrm{Cl}$ & $\mathrm{p}$ value \\
Right stroke work index & 1.12 & $1.03-1.21$ & 0.007 \\
DLCO & 0.94 & $0.87-1.01$ & 0.072 \\
\hline
\end{tabular}

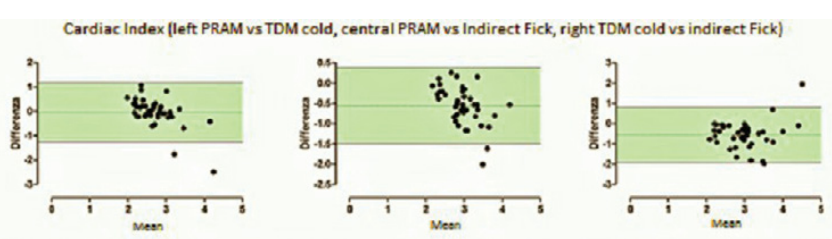

Pulmunary vascular resistancies (left PRAM vs TDM cold, central PRAM vs indirect Fick, right TOM cold vs indirect Fick)
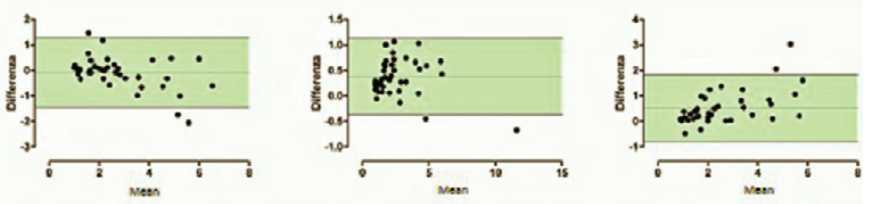

Systemic vascular resistancies (left PRAM vs TDM cold, central PRAM vs Indirect Fick, right TDM cold vs indirect Fick)
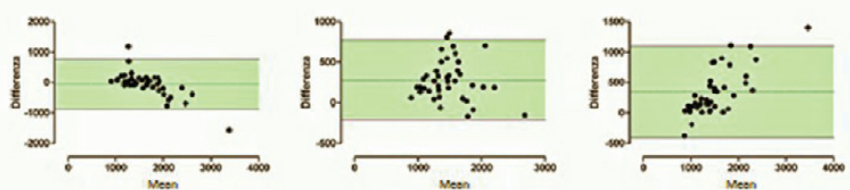

Central line represents mean difference between the two measures; upper and lower line is $95 \% \mathrm{Cl}$ assessed through Bland-Altmann analysis. Systolic pulmonary arterial pressure and TAPSE from echocardiography, forced vital capacity, total lung capacity, both absolute and alveolar volume adjusted Carbone oxide lung diffusion (DLCO and DLCO/VA) from pulmonary function tests, blood tests parameters, nailfold videocapillaroscopy scleroderma patterns were recorded. Univariate and multivariate logistic regression analysis identified variables correlating with $\mathrm{RHC}$ diagnosed PAH: a scoring system was then created, giving 1 point for value satisfying cut-off level.

Results: PRAM showed concordance with RHC estimate of $\mathrm{Cl}$ and SVR within 95\% interval confidence; 14 patients were diagnosed with PAH (mean age $64,4 \pm 9,3$, mean disease duration 10.5 years, ISSc $61,5 \%$ ). Multivariate logistic regression analysis showed DLCO (cut off value $47 \%$ obtained through ROC curve analysis, $\mathrm{p}=0.004$ ) and $\mathrm{RCPI}$ (cut off value 0.12 Watt obtained through ROC curve analysis, $\mathrm{p}<0.001$ ) as the most highly $\mathrm{PAH}$-associated variables. When combining these two variables in the scoring system, patient with score $=0$ (DLCO $<47 \%$ and $\mathrm{RCPI}<0,12$ Watt), score $=1$ (DLCO $<47 \%$ or $\mathrm{RCPI} \geq 0,12$ Watt) and score $=2(\mathrm{DLCO}<47 \%$ and $\mathrm{RCPI} \geq 0,12)$ were $0 \%, 21.4 \%$ and $78.6 \%$ of the $\mathrm{PAH}$ population respectively.

Conclusions: PRAM is comparable to RHC in detecting haemodynamic parameters such as SVR and Cl. The scoring system combining DLCO and RCPI, obtained with non-invasive tools, could offer the possibility of detecting $\mathrm{PAH}$ patients with a high specificity.

Disclosure of Interest: None declared

DOI: 10.1136/annrheumdis-2017-eular.5817

\section{FRI0385 JOINT MANIFESTATIONS IN PATIENTS DIAGNOSED WITH IDIOPATHIC INFLAMMATORY MYOPATHY: MULTICENTER REGISTRY}

D.C. Hernández Flórez ${ }^{1}$, L. Valor ${ }^{1}$, J. Martinez ${ }^{1}$, B. Joven ${ }^{2}$, L. Nuno ${ }^{3}$, C. Larena ${ }^{4}$, I. Llorente ${ }^{5}$, C. Barbadillo ${ }^{6}$, P. Garcia de la Pena ${ }^{7}$, L. Ruiz ${ }^{8}$, H. Moruno ${ }^{9}$, T. Cobo $^{10}$, R. Almodovar ${ }^{11}$, L. Lojo ${ }^{12}$, M. Garcia $^{13}$,

F. Lopez-Longo ${ }^{1} .{ }^{1} \mathrm{H}$. Gregorio Maranon; ${ }^{2} \mathrm{H} .12$ de Octubre; ${ }^{3} \mathrm{H} . \mathrm{La}$ Paz; ${ }^{4}$ H.Ramon y cajal; ${ }^{5}$ H.La Princesa; ${ }^{6}$ H.Puerta de Hierro; ${ }^{7}$ H.Norte Sanchinarro; ${ }^{8}$ H.Nino Jesus; ${ }^{9}$ H.Principe de Asturias, Madrid; ${ }^{10}$ H.Infanta Sofia, Asturias; ${ }^{11}$ H.F.Alcorcon; ${ }^{12}$ H. Infanta Leonor; ${ }^{13}$ InMusc, Madrid, Spain

Background: Idiopathic inflammatory myopathies (IIM) comprise a heterogeneous group of autoimmune conditions. Joint involvement can be considered to be part of the IIM systemic manifestations, together with a possible gastrointestinal, cardiovascular and/ or pulmonary involvement. Although articular involvement in the IIM has been described as variable and non-specific with a chronic course it might be an early symptom of dermatomiositis in up to $30 \%$ of cases and in those patients with overlap syndromes.

Objectives: To evaluate and to identify joint manifestations in IIM patients.

Methods: We evaluated a cohort of 479 patients that included 12 hospitals in the Community of Madrid belonging to the IIM registry of the Society of Rheumatology of Madrid (SORCOM-REMICAM) with diagnosis from January 1980 to December 2014. All patients were diagnosed of IIM according to Bohan and Peter criteria (1). The presence of arthralgia and arthritis was considered. IIM were classified as dermatomyositis (primary and secondary dermatomiositis) (DM) and polymyositis (PM) including the rest of the patients (classification I). Also, IIM were classified (II) as primary polimiositis (PPM), primary dermatomyositis (PDM), overlap syndrome (OSd), juvenile myopathies (JM), cancer-associated myopathies (CAM), autoimmune necrotizing myopathy and inclusion body myositis (these were grouped as other myositis; OM).

Results: We found 70 (18\%) patients with acute arthritis (<6 weeks), 74 (19\%) patients with chronic arthritis ( $>6$ weeks) and $245(65 \%)$ patients without any joint manifestations. Using the Tanimoto et al. criteria (1), the presence of erosive arthritis was observed in $149 / 479(38.3 \%)$ of the patients. When comparing the joint manifestations in the PM and DM groups ( $n=250,52.2 \%$ vs. $n=229,47.8 \%$ ) no statistically significant differences were observed. However, assessing joint manifestations according to classification II, we observed that the highest prevalence was found in the OSd group, followed by the PDM group $(p=0.0001)$. The group with less joint manifestations was JM compared to OSd and PDM (Table 1.

Table 1: Joint manifestations in IIM according to classification II

\begin{tabular}{|c|c|c|c|c|}
\hline & Comparison & en groups & Frequency & $\mathbf{P}$ \\
\hline $\begin{array}{c}\text { Joint } \\
\text { manifestations }\end{array}$ & OSd vs. & $\begin{array}{c}\text { PPM } \\
\text { PDM } \\
\text { JM } \\
\text { CAM } \\
\text { OM }\end{array}$ & $\begin{array}{l}90 \% \text { vs. } 54 \% \\
90 \% \text { vs. } 71 \% \\
90 \% \text { vs. } 45 \% \\
90 \% \text { vs. } 49 \% \\
90 \% \text { vs. } 58 \%\end{array}$ & 0,0001 \\
\hline Arthralgias & OSd vs. & $\begin{array}{c}\text { PPM } \\
\text { PDM } \\
\text { JM } \\
\text { CAM }\end{array}$ & $\begin{array}{l}90 \% \text { vs. } 54 \% \\
90 \% \text { vs. } 71 \% \\
90 \% \text { vs. } 44 \% \\
90 \% \text { vs. } 49 \%\end{array}$ & 0,0001 \\
\hline Arthritis & OSd vs. & $\begin{array}{c}\text { PPM } \\
\text { JM } \\
\text { CAM } \\
\end{array}$ & $\begin{array}{l}90 \% \text { vs. } 34 \% \\
90 \% \text { vs. } 28 \% \\
90 \% \text { vs. } 33 \% \\
\end{array}$ & 0,0001 \\
\hline
\end{tabular}

Conclusions: The presence of joint manifestations associated with IIM in our cohort is higher compared to other studies described in the literature so far and 
emphasize the importance of an accurate joint examination in these patients. The OSd group showed more joint manifestations which might be explained by the coexistence of SLE and MCTD patients in this group. Currently, no association between the clinical subtypes of IIM, overall, these results are encouraging and suggest that joint assessment in follow up may be helpful in differentiating subtypes of IIM.

References:

[1] Bohan A, Peter JB. N Engl J Med 1975 Feb 13;292(7):344-7.

Disclosure of Interest: None declared

DOI: 10.1136/annrheumdis-2017-eular.7051

\section{FRI0386 THE VALIDATION OF THE SCLERODERMA HEALTH ASSESSMENT QUESTIONNAIRE IN TURKISH SPEAKING PATIENTS WITH SYSTEMIC SCLEROSIS}

D. Temiz Karadag ${ }^{1}$, F. Karakas ${ }^{2}$, S. Tekeoglu ${ }^{1}$, O. Ozdemir Işik ${ }^{1}$, A. Yazici ${ }^{1}$, A. Cefle ${ }^{1} .{ }^{1}$ Rheumatology; ${ }^{2}$ Internal Medicine, Kocaeli University Medicine School, Kocaeli, Turkey

Background: The Health Assessment Questionnaire (HAQ) is one of the main instruments for assessing disability in rheumatic diseases.The Scleroderma $\mathrm{HAQ}$ (S-HAQ) combines the HAQ with five systemic sclerosis (SSc) related visual analogue scales for Raynaud's phenomenon (RP), digital ulcers, digestive symptoms, pulmonary symptoms, and overall disease severity.

Objectives: To perform cross-cultural adaptation and validation of the S-HAQ in patients with SSc.

Methods: Sixty patients who fulfilled the 2013 ACR/EULAR Classification Criteria for Systemic Sclerosis ACR criteria for SSc were were recruited.Fifty three percent of the patients fulfilled the criteria for limited systemic sclerosis (IcSSc).We evaluated test-retest reliability using the intraclass correlation coefficient (ICC); known-groups construct validity by stratifying patients according to severe organ involvement; and convergent validity using Spearman's correlation with mental and physical components of Short Form 36 version 2 (SF36v2).

Results: Eighty percent of the patients were female and the median age was 56 years old.Finger tip ulcers were observed in 16 patients $(26.7 \%)$ and severe organ involvements were detected as intersititial lung disease in $32(53 \%)$ and pulmonary hypertension in $5(8 \%)$ of the patients. HAQ Disability Index (HAQ-DI), digestive VAS, pulmonary VAS, digital ulcer VAS, Raynaud's phenomenon VAS and overall disease severity VAS demonstrated high reliability (ICCs $=0.819$ 0.908). The HAQ-DI showed higher correlation coefficients with physical function and role physical components of physical-related scores $(r=-0.537$ and -0.453 , respectively); social function and role emotional components of mental-related dimensions ( $r=-0.470$ and -0.328 , respectively) in the SF-36. Two among five SSc related VAS (VAS digital ulcer and VAS overall disease severity) were highly correlated with HAQ-DI ( $r=0.527$ and 0.481 respectively). The instruments could not discriminate between severe organ involvements and both subsets of SSc.

Table 1. Correlation of HAQ-DI with physical and mental components of SF-36 and, SSc related VAS scores

\begin{tabular}{lccc}
\hline $\mathrm{N}=60$ & Mean \pm SD and/or $\%$ & Correlation Coefficient & $p$-value \\
\hline Physical function & $55.4 \pm 23.83$ & -0.537 & 0.000 \\
Role physical & $33.82 \pm 42.67$ & -0.453 & 0.001 \\
Body pain & $53.82 \pm 28.84$ & -0.290 & 0.041 \\
General health & $39.9 \pm 18.43$ & -0.295 & 0.037 \\
Vitality & $41.08 \pm 18.39$ & -0.286 & 0.044 \\
Social function & $62.25 \pm 24.49$ & -0.470 & 0.001 \\
Role emotional & $49.02 \pm 32.9$ & -0.328 & 0.02 \\
Mental health & $61.12 \pm 18.39$ & -0.295 & 0.04 \\
VAS digestive & $0.39 \pm 0.65$ & 0.304 & 0.028 \\
VAS pulmonary & $0.64 \pm 0.79$ & 0.380 & 0.005 \\
VAS RP & $0.67 \pm 0.74$ & 0.354 & 0.01 \\
VAS digital ulcer & $0.62 \pm 0.94$ & 0.527 & 0.000 \\
VAS overall severity & $1.14 \pm 0.97$ & 0.481 & 0.000 \\
\hline
\end{tabular}

Conclusions: The Turkish version of the S-HAQ meet the requirements of reproducibility and validity.More studies are needed to examine the capacity of these instruments to discriminate between severe involvements and disease subsets. This study forms the basis for future studies to evaluate the properties of $\mathrm{S}-\mathrm{HAQ}$ in Turkish speaking SSc patients more extensively.

\section{References:}

[1] R Hesselstrand et al, Psychometric properties of the Swedish version of the Scleroderma Health Assessment Questionnaire and the Cochin Hand Function Scale in patients with systemic sclerosis, Scandjrheumatol Vol. 42, Iss. 4,2013.

Disclosure of Interest: None declared

DOI: 10.1136/annrheumdis-2017-eular.3289

\section{FRI0387 ANTI-RNA POLYMERASE III SUBSET OF SCLERODERMA PATIENTS: A MONOCENTRIC STUDY}

E. Pigatto, M. Ferranti, M. Favaro, P. Polito, E. Zanatta, P. Galozzi, M. Tonello, L. Punzi, F. Cozzi. University of Padova, Padova, Italy

Background: Systemic sclerosis (SSc) is an autoimmune disease characterized by heterogeneous clinical features and variable disease course. Specific antinuclear antibodies (ANA) identify different clinical subsets and are very useful in defining the prognosis of the patients. Recently, the anti-RNA polymerase III antibodies (anti-RNAP) were defined as the third specific ANA of SSc, together with anti-centromere (ACA) and anti-topoisomerase I (anti-Scl70).

Objectives: The aim of this study was to analyze the clinical picture of a group of SSc patients with anti-RNAP in a monocentric cohort of patients and to compare it with other subsets of patients with different specific ANA. In particular, the visceral involvement and the concomitant malignancies were investigated.

Methods: Among the SSc patients referring to the Rheumatology Unit of Padova University, 49 cases with anti-RNAP specific ANA were considered. Patient's demographics data and major clinical manifestations were compared with those of 50 ACA-positive and 52 anti-Scl70-positive patients. The skin score and the presence of digital ulcers, arthritis, interstitial lung disease (ILD), cardiac involvement including pulmonary hypertension, gastrointestinal disease (GI) and scleroderma renal crisis (SRC) were assessed. The presence and type of concomitant malignancies were also evaluated.

Results: $53 \%$ of anti-RNAP-positive patients had a diffuse cutaneous form of SSc. The skin score was significantly higher than in ACA-positive patients $(p<0.001)$. Digital ulcers were active in $73.5 \%$ of cases, without significant difference with the other two subsets. Arthritis was found in $20.4 \%$ of cases in comparison to $4 \%$ in ACA-positive patients $(\mathrm{p}<0.02)$. Regarding visceral involvements, $\mathrm{Gl}$ disease was the most common manifestation in all subsets; SRC was observed in $24.4 \%$ of anti-RNAP-positive patients and was significantly more frequent in comparison to ACA-positive $(p<0.005)$; ILD showed a prevalence of $42.8 \%$ in anti-RNAP subset, lower than in anti-Scl70-positive $(p<0.05)$ and higher than in ACA-positive patients $(p<0.01)$; cardiac involvement was observed in $32.6 \%$ of anti-RNAP-positive patients, without significant difference with the other subsets. Malignancies were found in $33(67.3 \%)$ anti-RNAP-, 18 (36\%) anti-Scl70- and $7(14 \%)$ ACA- positive patients. The most common cancer-sites were breast $(36 \%)$, lung (14\%) and colon (10\%). The risk of developing cancer was higher in anti-RNAP-positive patients than in other subsets (OR:6.35).

Conclusions: Our data demonstrated that anti-RNAP specific antibodies can identify a subset of SSc patients characterized by a severe clinical picture, with a high prevalence of diffuse cutaneous form, SRC, cardiac involvement and ILD. These patients showed also an elevated risk of developing cancer. Our results were consistent with recent published papers on this topic $(1,2)$.

\section{References:}

[1] Terras S et al. RNA polymerase III autoantibodies may indicate renal and more severe skin involvement in systemic sclerosis. Int J Dermatol 2016; 55:882-5.

[2] Lazzaroni MG et al. Malignancies in patients with anti-RNA Polymerase III antibodies and Systemic Sclerosis: analysis of the EULAR Scleroderma Trials and Research Cohort and possible recommendations for screening. J Rheumatol 2017; pii: jrheum.160817.

Disclosure of Interest: None declared

DOI: 10.1136/annrheumdis-2017-eular.3292

\section{FRI0388 LONG-TERM TREATMENT WITH RITUXIMAB IN SYSTEMIC SCLEROSIS PATIENTS: UPDATE OF OUR CLINICAL EXPERIENCE}

F. Lumetti, M. Colaci, C. Esposito, E. Cocchiara, C. Vacchi, A. Spinella, G. Tiengo, D. Giuggioli, C. Ferri. Scleroderma Unit, Chair of Rheumatology, University of Modena and Reggio Emilia, Policlinico of Modena, Modena, Italy

Background: Systemic sclerosis (SSc) is an immune-mediated disorder characterized by abnormal fibrosis and diffuse microangiopathy with skin and internal organ involvement. The treatment of SSc represents a great clinical challenge because of the complex disease pathogenesis including vascular, fibrotic, and immune T- and B-lymphocyte-mediated alterations. Therefore, SSc should be treated by combined or sequential therapies according to prevalent clinico-pathogenetic phenotypes. Some preliminary data suggest that rituximab (RTX) may be usefully employed in SSc patients.

Objectives: The present study aimed to evaluate the efficacy of RTX in our SSc patients' series as well as the long-term effects of this treatment.

Methods: A series of $15 \mathrm{SSc}$ patients (M/F 6/9, mean age $52.7 \pm 17.9 S D$ years, mean disease duration $10.3 \pm 7.1 \mathrm{SD}$ years, $\mathrm{L} / \mathrm{D}$ cutaneous subsets $5 / 10$ ) were treated with one or more cycles of RTX (4 weekly infusions of $375 \mathrm{mg} / \mathrm{m}^{2}$ ). In all patients RTX was repeated every 6 months for a total of 2-6 cycles. Patients' clinical-serological evaluation, including the self-evaluation of quality of life by means of $\mathrm{HAQ}$ and visual analogical scale (VAS) assessment, was performed every 6 months for a mean follow-up period of $42 \pm 24$ SD months.

Results: After the first 6 months following RTX treatment the extent of skin sclerosis measured with modified Rodnan skin score (mRSS) significantly improved (from $17.3 \pm 10.4$ to $13.4 \pm 7.6 ; p<.01$ ), and remained stable at the end of the follow-up $(13.3 \pm 8.1 ; p=.009)$. The usefulness of RTX on skin sclerosis was more evident in patients with diffuse cutaneous SSc $(n=10)$ showing a significant decrease of mRSS after the first 6 months (from $24.2 \pm 5.1$ to $18.1 \pm 4.7 ; p=.006$ ) and at the end of the follow-up period $(18.0 \pm 6.0 ; p=.005)$. Similarly, a valuable improvement of other cutaneous manifestations, namely hypermelanosis $(12 / 12$ pts), pruritus (11/13 pts), and calcinosis ( $3 / 6$ pts) was observed. Moreover, arthritis revealed particularly responsive to RTX treatment leading to a clear-cut reduction of swollen and tender joints in 12/13 patients; while lung fibrosis detected in 